\title{
Oral health gap in rich and poor areas is worsening
}

The gap in dental decay rates in children between those from the most and least deprived areas in Scotland appears to be growing, according to the latest National Dental Inspection Programme Report. ${ }^{1}$

The official NHS report for 2017-18 published on 23 October 2018 contains results from a survey of 16,814 primary one (P1) pupils and found $86 \%$ of those children in Scotland's least deprived areas had no obvious signs of decayed, missing, or filled milk teeth compared with $56 \%$ in the most deprived areas.

This gap has grown by three percentage points compared with the previous year's (2016-17) results. In addition, the average number of decayed, missing or filled teeth was 0.45 for children in the most affluent areas, but 1.92 in the poorest places.

However, the overall oral health of Scotland's children was continuing to improve with $71 \%$ of them having no obvious signs of decay, representing an improving trend that has risen 1994 when the rate was only $38 \%$ and which increased by two percentage points since 2016-17.

The BDA said the Scottish Government needed to do more to tackle oral health inequalities, given the stark differences across Scotland such as the percentage of P1 children free from dental decay in Dumfries and Galloway being 67\%, while the rate was $84 \%$ for children in Orkney.

The union welcomed the extension of the Childsmile (http:// www.child-smile.org.uk/) programme - the national oral health improvement programme for children in Scotland - but urged the Scottish Government to do more and have a greater focus on prevention.

Robert Donald, Chair of the BDA's Scottish Council said: 'Dentists have applauded the progress secured through Childsmile, but this data is a stark reminder that the Scottish Government cannot rest on its laurels.

'Scotland's oral health gap had shown signs of closing, and we must ensure hard-won progress is not undone. Ministers now need to go further and faster to tackle the scandal of these deep and persistent health inequalities.

A Scottish Government spokesperson said: 'We welcome that the proportion of children with no obvious decay experience has increased to $71 \%$ in 2018, compared with $58 \%$ in 2008 .

'We want to see these positive trends continue by tackling health inequalities in children. This is why children living in our most deprived areas are entitled to receive fluoride varnish applications at nursery or school.

'We have also extended the Childsmile Programme to all nursery and primary one and two children in the most deprived communities across Scotland,

1. NHS National Services Scotland. National Dental Inspection Programme (NDIP) 2018 (23 October 2018). https://www.isdscotland.org/Health-Topics/Dental-Care/Publications/2018-10-23/2018-10-23-NDIP-Report.pdf (accessed on 5 November 2018).

\section{Oral health left out of global health goals}

Oral health has been omitted from the latest United Nations (UN) Sustainable Development Goals (SDGs) to improve healthcare across the globe that were agreed recently in Brussels.

During a meeting of the UN's General Assembly held on 27 September 2018, world leaders and policy makers debated the global impact of noncommunicable diseases but oral health was not part of the debate and was not included in the final Political Declaration that committed signatories to take action on its many clauses.

Heads of state and government committed to 13 new steps to tackle non-communicable diseases (NCDs) including cancers, heart and lung diseases, stroke, and diabetes, and to promote mental health and well-being.

World leaders agreed to take responsibility themselves for their countries' effort to prevent and treat NCDs. They also agreed that these efforts should include laws and fiscal measures to protect people from tobacco, unhealthy foods, and other harmful products, for example, by restricting alcohol advertising, banning smoking, and taxing sugary drinks.

They committed to implement a series of World Health Organisation (WHO) recommended policies to prevent and control NCDs, such as public education and awareness campaigns to promote healthier lifestyles, vaccinating against HPV virus to protect against cervical cancer and treating hypertension and diabetes.

WHO has estimated that implementing all these policies could generate US\$ 350 billion in economic growth in low and lower-middle융 income countries between now and 2030.
Other specific commitments focus on halting the rise of childhood obesity, promoting regular physical activity, reducing air pollution and improving mental health and wellbeing.

The political declaration also called on food manufacturers to take several actions, including reformulating products to reduce salt, free sugars and saturated and industrially produced trans fats, using nutrition labelling on packaged food to inform consumers, and restricting the marketing of unhealthy foods and beverages to children.

Dr Tedros Adhanom Ghebreyesus, Director-General of the WHO, said: 'Today, world leaders have taken a set of landmark steps to beat NCDs. These add up to a historic opportunity to promote health, save lives, and grow economies.'

In October 2018, the International Association of Dental Research (IADR) joined the NCD Alliance - a global network of more than 2,000 organisations in 170 countries across the NCD community - to help boost the profile of oral health and better articulate the case for the inclusion of oral health in the fight against NCDs.

IADR Chief Executive Officer Christopher Fox said: 'The IADR is pleased to join the NCD Alliance in the fight against NCDs and support the inclusion of oral health in these important global policy discussions.'

To read more about this topic, turn to David Croser's Opinion Article in this issue entitled 'Oral health waits another seven UN years' (BDJ 2018; 225: 927-929, DOI: 10.1038/sj.bdj.2018.1021). 